\title{
Questes
}

\section{En guise d'avant-propos : les communautés ont quand même une histoire}

Joseph Morsel

\section{(2) OpenEdition}

1 Journals

\section{Édition électronique}

URL : http://journals.openedition.org/questes/4324

DOI : 10.4000/questes.4324

ISSN : 2109-9472

Éditeur

Les Amis de Questes

\section{Édition imprimée}

Date de publication : 6 mai 2016

Pagination : 1-14

ISSN : 2102-7188

\section{Référence électronique}

Joseph Morsel, «En guise d'avant-propos

les communautés ont quand même une histoire ", Questes [En ligne], 32 | 2016, mis en ligne le 10 mai 2016, consulté le 24 septembre 2020. URL : http://journals.openedition.org/questes/4324 ; DOI :

https://doi.org/10.4000/questes.4324 


\title{
En guise d'avant-propos : les communautés ont quand même une histoire
}

\author{
Joseph MORSEL \\ LaMOP - Université Paris 1 Panthéon-Sorbonne
}

Mourir pour la patrie est la meilleure preuve ontologique de l'existence de celle-ci. La seule, peut-être ${ }^{1}$.

«Faire communauté »: l'objet ainsi désigné soulève heureusement plusieurs problèmes historiques fondamentaux, liés pour l'essentiel à l'enjeu même de l'histoire, conçue comme science du changement social. Le verbe «faire » attire en effet l'attention sur l'existence d'un processus ou d'un acte d'institution, qui crée un avant et un après, donc un changement, une transformation. Ce changement provoqué peut lui-même être examiné à deux niveaux (au moins) : celui, phénoménal, de telle ou telle communauté, dont on examine la genèse, le passage du non-encorecommunautaire au communautaire (au risque, toujours, de la téléologie) ; ou alors celui, plus largement historique, de l'apparition ou multiplication de communautés dans le paysage social de l'Occident médiéval, par exemple sur le mode de ce que Pierre Michaud-Quantin a appelé «mouvement communautaire dans le Moyen Âge latin », corrélatif de l'extrême productivité des auteurs des $\mathrm{XII}^{\mathrm{e}}-\mathrm{XIII}$ e siècles, avant même la diffusion de l'aristotélisme ${ }^{2}$.

\footnotetext{
${ }^{1}$ Jorge Semprun, L'Écriture ou la vie, 1994.

2 Pierre Michaud-Quantin, Universitas. Expressions du mouvement communautaire dans le Moyen Âge latin, Paris, J. Vrin, 1970.
} 
Cette manière d'attirer l'attention sur l'historicité de la communauté mérite d'être soulignée ne serait-ce que parce qu'elle distingue bien la démarche adoptée dans le présent dossier de deux autres approches. La première distinction concerne un autre dossier «communautaire » présenté il y a une dizaine d'années par un autre groupe de doctorants (mais pas seulement médiévistes), intitulé «Instituer la communauté ${ }^{3} »$. L'apparente proximité des formulations ne doit cependant pas tromper: ce qui importait alors, explicitement, était le verbe «instituer», dans son acception juridico-institutionnelle, à l'opposé (assumé) d'une perspective plus sociologique (et donc classique) centrée sur la construction du lien social.

L'écart des approches est d'autant plus visible que la plupart des cas traités dans le présent dossier concernent des «communautés » précisément non instituées : dans l'ordre chronologique apparaissent ainsi les interfaces socioculturelles au sein des États latins d'Orient $\left(\mathrm{F}\right.$. Besson $\left.^{4}\right)$, les lecteurs d'un texte qualifié d' « hérétique » $\left(\right.$ É. Pinel $\left.{ }^{5}\right)$, un assemblage de fidèles qu'on tente de former autour du roi à Troyes (C. Rager ${ }^{6}$ ), les imprimeurs vénitiens (C. Kikuchi $\left.{ }^{7}\right)$. Seules deux contributions traitent de communautés effectivement instituées, en l'occurrence urbaines : la communauté d'habitants d'Estella (A. Giunta ${ }^{8}$ ) et celle de Valenciennes ( $\mathrm{S}$. Huart ${ }^{9}$ ) - et parmi celles-ci, seule la contribution sur Estella traite expressément du problème de la fondation

${ }^{3}$ Instituer la communauté, dir. Paulin Ismard et Émilie Rosenblieh, dans Hypothèses 2005. Travaux de l'École doctorale d'Histoire de l'Université Paris 1, vol. 9, n ${ }^{\mathrm{o}} 1$, 2006, p. 105-161.

${ }^{4}$ Voir l'article de Florian Besson dans ce volume, p. 35-50.

${ }^{5}$ Voir l'article d'Élodie Pinel dans ce volume, p. 69-83.

${ }^{6}$ Voir l'article de Cléo Rager dans ce volume, p. 123-138.

${ }^{7}$ Voir l'article de Catherine Kikuchi dans ce volume, p. 139-156.

${ }^{8}$ Voir l'article d'Alexandre Giunta dans ce volume, p. 51-67.

${ }^{9}$ Voir l'article de Stéphanie Huart dans ce volume, p. 85-101. 
d'une communauté. Par conséquent, «faire la communauté » n'a nullement été examiné sous le seul angle de l'institution.

L'autre distinction significative entre ce dossier et la manière dont a longtemps été (et est encore souvent) traité le problème de la «communauté » réside précisément dans l'historicité qui lui est implicitement prêtée. Cela signifie que le mot «communauté »n'est pas ici - et ne peut être - employé pour n'importe quelle forme collective ou de coprésence humaine, comme c'est trop souvent le cas (par exemple pour les bandes de chasseurs préhistoriques ou des groupements de cabanes du haut Moyen Âge): c'est ainsi parce qu'il considère la communauté avant tout comme une forme de peuplement groupé que l'historien britannique Rodney Hilton estime pouvoir en rencontrer dès l'âge du fer, voire à la fin de l'âge du bronze (c'est-à-dire vers 1000 avant notre ère $\left.{ }^{10}\right)$. La communauté devient dès lors, pour reprendre les mots d'Alain Guerreau, une «entéléchie a-historique, dont la toute-puissance défie les siècles... et les modes de production ${ }^{11} »$.

Le risque est grand, dès lors, de faire remonter la forme communautaire aux origines obscures de l'humanité - aux deux sens du terme. D'une part en effet, sociologues et anthropologues ont longtemps considéré la communauté comme une sorte d'inverse de la société, dont elle formerait la préhistoire: chez le précurseur Ferdinand Tönnies comme chez la plupart des anthropologues, notamment anglo-saxons, jusqu'aux années $1960^{12}$, la communauté correspondait ainsi à un état

${ }^{10}$ Rodney Hilton, Les Mouvements paysans du Moyen Âge et la révolution anglaise de 1381 [1973], trad. fr. Paris, Flammarion, 1979, p. 31.

11 Alain Guerreau, Le Féodalisme. Un horizon théorique, Paris, Le Sycomore, 1980, p. 86. Sur les difficultés d'emploi que présente pour l'historien la notion de «communauté », je me permets de renvoyer à ma notice «Communauté », dans : Dictionnaire de l'historien, dir. Jean-François Sirinelli et Claude Gauvard, Paris, PUF, 2015, p. 107-110.

12 Ferdinand Tönnies, Gemeinschaft und Gesellschaft, Berlin, 1887, trad. fr. 
antérieur à la société (et à l'histoire), caractérisant tout particulièrement les sociétés préindustrielles et/ou non occidentales. Mais d'autre part, c'est aussi l'humanité de l'homme qui est en jeu dans l'exigence qu'ait existé de tout temps une forme au moins proto-sociale : comment rendre compte, sans cela, de l'essence de l'homme (dès lors qu'elle n'est plus rapportée à la Création), un homme défini par Aristote comme «animal communautaire » (koinonikon zôon) avant même que d'être un politikon zôon - c'est-à-dire moins un «animal politique » que, dans le contexte grec, un « animal proprement social»?

Cela ne signifie évidemment pas qu'il n'a existé des communautés que dans l'Occident du second Moyen Âge (schématiquement à partir du XII siècle, correspondant à ce que Robert Fossier appelait « encellulement», ou au «mouvement communautaire» de Pierre Michaud-Quantin qui attire l'attention sur les réflexions théologicojuridiques permettant désormais de concevoir le principe de «personnes morales ») : simplement, l'on n'a absolument rien à gagner à se contenter d'un usage flou du mot, parce qu'il interdit précisément de saisir la spécificité des formes sociales qui résultent des processus examinés. Un tel effort de précision conceptuelle et d'historicisation pose d'ailleurs le problème classique du balancement entre vocabulaire indigène (où existent communitas et divers équivalents vernaculaires) et conceptualisation scientifique - deux aspects cependant (et

Communauté et société, Paris, PUF, 1946 ; on remarquera d'ailleurs en passant que « faire la communauté » est strictement antithétique de la position de Tönnies puisque pour lui, la communauté désigne un groupement naturel et organique, auquel on appartient automatiquement, à l'inverse de la société fondée sur le consentement, le contrat (social), l'adhésion volontaire... Sur la recherche anglo-saxonne, cf. John W. Cole, «Anthropology comes Part-Way Home: Community Studies in Europe », Annual Review of Anthropology, vol. 6, 1977, p. 349-358, ainsi que Cherry Schrecker, La Communauté. Histoire critique d'un concept dans la sociologie anglosaxonne, Paris, L'Harmattan, 2006. 
malheureusement) peu abordés dans le présent dossier mais qui, il est vrai, présentent de redoutables difficultés.

Néanmoins, posant ainsi le problème sous l'angle du «faire », celui-ci implique un authentique travail d'historien, puisque la question implique de faire apparaître l'historicité du phénomène social et des usages conceptuels ainsi que la pertinence des échelles d'analyse - les «communautés» ici envisagées allant des États (latins) au métier (vénitien), en passant par les divers groupes de lecteurs du Miroir des simples âmes et les villes (d'Estella, Valenciennes et Troyes), pour ce qui est des échelles spatiales, et de quelques siècles à quelques années pour ce qui est des échelles temporelles.

Puisqu'il convient de clarifier ce à quoi l'on prétend avoir affaire quand on parle de «communauté », et que suggèrent (implicitement, on l'a dit) les contributions du dossier, le plus simple est probablement de partir d'une définition négative - c'est-à-dire de ce que n'est pas une «communauté »: c'est souvent plus facile et plus efficace. Cette définition négative peut être opérée, quant à elle, de manière abstraite ou par opposition à une autre forme historique. Dans le premier cas, on pourra considérer ce qu'une communauté $n^{\prime} e s t$ pas ${ }^{13}$ : elle n'est pas une forme nécessairement agglomérée; une forme parentale; une forme centripète (au contraire, elle se démarque par rapport à l'extérieur) ; une forme qui se confond avec l'ensemble des personnes présentes dans le lieu ou le territoire considéré ; une forme nécessairement d'interconnaissance.

Ces critères correspondent d'ailleurs à l'écart qu'il me semble pouvoir observer entre une paroisse et une communauté - ce qui me conduit à rejeter la notion de «communauté paroissiale » pour la période

${ }^{13}$ Ces divers critères sont explicités dans ma notice mentionnée supra, n. 4, à laquelle je me contente donc de renvoyer. 
médiévale $^{14}$. Le point-clé me semble résider dans le fait que la paroisse ne se définit pas par les rapports des paroissiens entre eux, mais par les rapports entre paroissiens et curé : celui-ci médiatise les rapports entre les paroissiens, y compris au sein des familles puisqu'il dote en théorie tout fidèle de nouveaux parents, baptismaux. Or, pour qu'il y ait communauté, il faut qu'il y ait rapport entre les membres (de la communauté) euxmêmes, mutuels (ou « horizontaux », sans que cela exclue cependant des inégalités de position), et non pas hiérarchisés et polarisés sur tel ou tel personnage.

Cette distinction n'est toutefois pas seulement pertinente pour saisir la nature de la paroisse mais aussi pour rendre compte de la situation des imprimeurs vénitiens présentée par C. Kikuchi, dans laquelle l'absence de toute communauté de métier (arte) est strictement corrélative du fait qu'on ne repère que des rapports interpersonnels entre les imprimeurs individuels et le pouvoir (auprès duquel ils se dénoncent d'ailleurs les uns les autres): qu'ils aient la même activité dans le même espace, voire même sur la base d'alliances matrimoniales, n'est donc pas du tout suffisant pour parler de «communauté ». Certes, dans ce cas précis, la question pourrait être déplacée du côté de l'absence d'institution - mais C. Kikuchi montre bien que s'il n'y a pas d'arte des imprimeurs, ce n'est pas faute d'intérêt des patriciens pour contrôler des activités artisanales (ce à quoi sert le système des arti) : c'est donc que la dynamique communautaire n'existe pas (encore).

${ }^{14}$ Cette distinction est au cœur de mon approche des «communautés d'habitants » médiévales : Joseph Morsel, " "Communautés d'installés". Pour une histoire de l'appartenance médiévale au village ou à la ville », EspacesTemps.net, mis en ligne le 11.11.2014, http://www.espacestemps.net/articles/ communautes-dinstalles/, ainsi que «La faucille et le goupillon? Observations sur les rapports entre communauté d'installés et paroisse dans l'Empire », dans Communautés d'habitants médiévales, dir. Joseph Morsel, à paraître (Paris, Publications de la Sorbonne, 2017). 
La question de l'absence d'institution qui pourrait brouiller la compréhension du phénomène ne se pose en revanche pas dans le cas de Troyes vers 1430 , où l'on observe remarquablement bien la distinction faite ci-dessus entre communauté et faisceau de liens polarisés. Car ce que montre C. Rager, c'est précisément l'absence d'une communauté (dite ici «politique »), c'est-à-dire l'échec (si tant est que cela ait été l'objectif) d'une conversion de la communauté d'habitants en communauté «politique », dû à l'existence d'une population divisée quant à la loyauté à avoir envers le dauphin ainsi qu'à une large continuité des élites municipales avant et après.

Par conséquent, ce qu'instituent les autorités (royales et/ou municipales) est précisément une forme centrée/focalisée sur le roi (par le serment de fidélité ${ }^{15}$ ), dans laquelle être «habitant » et être citadin sont placés sur le même $\operatorname{plan}^{16}$, et au sein de laquelle les rapports entre membres de la communauté sont conçus moins comme une collaboration (mutuelle) que comme une surveillance et une incitation à la délation (bref une collaboration entre chacun et le pouvoir - que le régime de Vichy peut nous permettre de nous représenter...). Rien de tout cela ne correspond à une communauté stricto sensu, pas plus que le «bien commun » à l'horizon du «bien du roi et de la ville »: non seulement le «bien commun » ou «commun profit » n'apparaît même pas dans les

15 Pierre Michaud-Quantin, Universitas..., op. cit., p. 235, avait déjà insisté sur le caractère non communautaire des serments de fidélité.

${ }^{16}$ Je fais référence ici au second article du serment cité par C. Rager, dans lequel les manans et habitans de la ville (c'est-à-dire les membres de la communauté d'habitants) sont traités comme les autres estans. Cette distinction fait un remarquable écho à la thématique développée en septembre 2005 lors d'un colloque du programme du LaMOP consacré aux Communautés d'habitants médiévales et qui portait précisément sur l'enjeu de l' « habiter » pour caractériser les communautés médiévales (cf. par exemple le titre de la contribution de Ludolf Kuchenbuch : «De la station à l'habiter ? Observations qualitatives et comparatives à partir de documents rhénans des VIII $-\mathrm{IX}^{\mathrm{e}}$ et $\mathrm{XII}^{\mathrm{e}}-\mathrm{XIII}^{\mathrm{e}}$ siècles »); sur tous ces aspects, je me permets de renvoyer aux travaux cité supra, n. 7 . 
extraits fournis, mais même cette notion s'avère, à bien y regarder, être une pure technique de justification pour un pouvoir seigneurial ${ }^{17}$.

Pourtant, les autorités tentent de définir une identité collective fondée à la fois sur un insigne à arborer et la définition d'un ennemi commun (l'insigne étant fixé sur les portes de la ville). Le cas de Valenciennes présenté par S. Huart suggère aussi que la solidarité des habitants, au-delà de leurs intérêts particuliers, pouvait être atteinte ou réactivée face à une menace extérieure. Mais dans les deux cas (Troyes et Valenciennes), on voit que ce facteur - correspondant au critère susmentionné de la démarcation par rapport à l'extérieur - ne semble pas (encore) le plus pertinent, le «patriotisme urbain » parfois évoqué par les historiens de la ville médiévale relevant peut-être plus du désir et du discours de ses maîtres (ou des croyances des historiens, à cause de nos représentations national[ist]es) que des représentations de la population...

La même question peut être posée à propos de mesures de démarcation face à des éléments internes à la communauté dont l'intégration communautaire pose problème, et qui sont de ce fait placés en position d' « étrangers de l'intérieur » : c'est ainsi que Thomas d'Aquin qualifie les juifs, vers 1270, de ceux qui foris sunt (il réactive ainsi une expression néo-testamentaire: Marc, IV, 11; 1 Corinthiens, V, 13 ; Colossiens, IV, 5 ; 1 Thessaloniciens, IV, 12 ; 1 Timothée, III, 7) alors même qu'il parle de juifs résidents, mise « hors espace » symbolique qui

${ }^{17}$ Sur les nombreuses ambiguïtés (et pas seulement incertitudes) de la notion de « bien commun», cf. d'une part Gemeinwohl und Gemeinsinn. Historische Semantiken politischer Leitbegriffe (Forschungsberichte der interdisziplinären Arbeitsgruppe „Gemeinwohl und Gemeinsinn“ der Berlin-Brandenburgischen Akademie der Wissenschaften, Band I), dir. Herfried Münkler, Harald Bluhm et Karsten Fischer, Berlin, Akademie Verlag, 2001 (concernant, pour le Moyen Âge, l'Allemagne, la France et l'Angleterre), ainsi que mon article à paraître, «Res publica et gemeiner nutz. Observations à partir de l'espace germanophone (XIV $-\mathrm{XV}^{\mathrm{e}}$ siècles) $»$, Quaestiones Medii Aevi Nova, 2016. 
prélude à des expulsions effectives ${ }^{18}$. Mais de telles expulsions sont également mises en œuvre par les autorités urbaines à Valenciennes de même que par les représentants du roi à Troyes : dans le premier cas, S. Huart voit dans les multiples bannissements auxquels on procède un moyen de «maintenir la société urbaine en un groupe soudé » (par la menace de cette sanction grave, par l'expulsion des éléments dangereux et par la valeur symbolique de l'exclusion physique/spatiale - à quoi l'on pourrait sans doute ajouter, sur la base de la lettre échangée entre les échevins de Valenciennes et ceux de Saint-Quentin, la question de l'image de robustesse présentée aux autres villes); les multiples bannissements «cassés » par le pouvoir comtal témoigneraient quant à eux de la concurrence entre deux pouvoirs qui, précisément, jouent sur les limites de la démarcation (une concurrence qu'on peut subodorer aussi à Estella, entre le roi et l'abbaye).

Dans le cas de Troyes, il s'agit d'exclure les supposés « traitres » au roi, sur la base de dénonciations - l'intérêt du cas troyen étant que ces expulsions se doublent d'excommunications (qui n'impliquent en tant que telles pas d'exclusion de la communauté d'habitants: elles excluent «seulement» de l'ecclesia, tant locale que globale). Cet usage «en creux » de l'excommunication, c'est-à-dire pour renforcer la cohésion du groupe par l'exclusion de certains éléments présentés comme nocifs ou dangereux, se rencontre par ailleurs dans le cas présenté par É. Pinel, qui insiste bien sur le fait que l'orthodoxie a besoin de l'hérésie pour se définir comme telle et se renforcer - d'où l'adoption des propositions faites par et autour de Monique Zerner, selon lesquelles l'hérésie existe avant tout dans l'œil des clercs, qui transforment ainsi en contre-modèles radicaux des personnes qui sont certes à la limite, voire éventuellement

${ }^{18}$ Dominique Iogna-Prat, «La terre sainte disputée », Médiévales, 41, 2001, p. 83112, notamment p. 106-109. 
déviantes, mais pas en-dehors de l'Église : le cas de Marguerite Porete est emblématique de cela, non seulement à cause de sa volonté de rénovation interne (et non pas de rupture), de sa qualification comme hérétique mais aussi de l'incertitude quant à la perception qui a été celle de sa position (les passages les plus critiquables du point de vue de la théologie sacramentelle n'apparaissent, semble-t-il, que dans les manuscrits les plus tardifs, un siècle après l'exécution de M. Porete...). Bref, d'une position qui pouvait tout aussi bien être considérée comme mystique et, au pire, à encadrer, on a fait une extériorité (d'autant que M. Porete est condamnée comme relapse).

Il n'est pas jusqu'au cas de la communauté d'Estella qui suggère une manipulation des démarcations internes par ceux qui prétendent dominer la communauté. A. Giunta montre en effet comment les « habitants » (vecinos) d'Estella sont d'abord constitués en communauté urbaine, vers 1090, en les positionnant face à tout « Navarrais ou prêtre de l'extérieur » (Navarrus vel presbiter de foras), et insiste sur le fait que cette fondation relève avant tout d'une volonté royale de fixer en un lieu des populations mobiles (attirées par le pèlerinage de Compostelle et/ou les débuts de la Conquista), donc dans une logique de contrôle des populations. Au milieu du XII ${ }^{\mathrm{e}}$ siècle, on assiste visiblement à une requalification de ceux qui sont «en-dehors » : c'est en effet le moment où les juifs sont déplacés vers l'extérieur tandis qu'un quartier navarrais est installé à côté du noyau urbain précédent, puis un autre quartier mixte (sive navarrus, sive alius). De ce fait, il n'est pas étonnant que le qualificatif des premiers habitants, francos, change de sens: de «migrant» initialement, il finit par signifier «habitant» (au sens plusieurs fois évoqué, à savoir un membre à part entière de la communauté). 
Le problème du rapport (c'est-à-dire, en fait, de la définition) de la communauté vis-à-vis de l'étranger est aussi ce qui caractérise les deux autres contributions (sur Venise et les États latins), à des échelles toutefois très différentes. À propos d'Estella, A. Giunta souligne que ce qui forme le soubassement de la communauté d'habitants est «l'usage exclusif des aires urbaines », en l'occurrence pour des activités artisanales, commerciales et agricoles : ceci correspond tout à fait à l'un des résultats du programme développé dans le cadre du LaMOP sur la «Formation des communautés d'habitants ${ }^{19}{ }$, qui nous a conduit à définir la communauté d'habitants comme «structure d'allocation aux feux de l'accès à des moyens de production (agraires, artisanaux, commerciaux), allocation réalisée graduellement dans l'espace, le temps et la population». À Venise, C. Kikuchi rappelle que c'est bien ce qui se passe dans le cas du système des «métiers» (arti), et que le «métier» des verriers fait précisément l'objet d'un soutien du pouvoir municipal à l'encontre des verriers forains.

Or rien de tout cela ne s'observe à propos des imprimeurs durant le siècle 1470-1570, en dépit de la très grosse production vénitienne et, possiblement (si l'on en croit Érasme), de sa mauvaise qualité. Au contraire, on l'a déjà signalé, on ne repère que des rapports interpersonnels entre le pouvoir et les imprimeurs individuels, qui se dénoncent mutuellement (comme à Troyes) et qui par ailleurs ne forment/fréquentent aucune confrérie ou scuola spécifique. Si l'on écarte une argumentation en termes d'attitude égoïste ou individualiste - qui ne pourrait nullement être considérée comme une explication: les comportements individuels récurrents, voire statistiquement réguliers,

19 Programme présenté en détail sur le site du LaMOP (http://lamop.univparis1.fr/spip.php?rubrique68) et dont les résultats vont être publiés dans le volume mentionné supra, n. 7. 
sont pour l'essentiel socialement déterminés, et de toute façon il resterait alors à expliquer pourquoi les imprimeurs seraient plus particulièrement égoïstes/individualistes -, la seule explication que, finalement, l'on peut retenir est celle que suggère la comparaison avec le cas de Strasbourg: l'importance des étrangers (avérée dans le cas des imprimeurs vénitiens).

Pourtant, à Estella, c'est justement l'importance des francos qui conduit à leur constitution par le roi en une communauté urbaine - mais (outre l'écart de quatre siècles !) c'est là que prend toute son importance l'observation d'A. Giunta à propos de la fondation de la communauté urbaine d'Estella comme moyen de fixer une population qui, pour l'essentiel, vient d'elle-même (et qui est elle-même «doublée » par la venue de Navarrais). Dans le cas de Venise (si on admet l'analogie strasbourgeoise), il s'agissait de faire venir des imprimeurs, ou du moins de faciliter leur installation - si bien que les plaintes des uns et des autres quant au non respect de leurs privilèges ne devraient guère être considérées que comme le revers de la grande liberté dont ils jouissaient. Où l'on retrouve de nouveau l'idée selon laquelle, loin du romantisme attaché à l'idée de communauté, celle-ci devrait en premier lieu être considérée comme un mode d'encadrement social.

C'est exactement ce que suggère aussi le cas des États latins d'Orient, dont F. Besson montre que l'historiographie classique, focalisée sur des communautés imaginaires et anachroniques, n'a pas pu comprendre, d'une part, que s'y est instaurée une communauté véritable et spécifique (le «monde commun», sur la base d'une acclimatation mutuelle), d'autre part que derrière cette construction de la communauté se jouait avant tout le problème de la domination sociale - bref que le problème-clé était justement de passer d'une conjonction de rapports interpersonnels (vassaliques, commerciaux, sacramentels) et de la préservation, de part et d'autre, des hiérarchies sociales (symbolisée par le 
jeu d'échecs, qui affronte deux sociétés identiques selon des règles connues et partagées par les deux côtés) à un ensemble communautaire.

Tout comme l'avait fait remarquer Reyna Pastor à propos de la Conquista $^{20}$, F. Besson montre qu'on ne doit pas se laisser prendre au piège des schématisations cléricales (qui binarisent les oppositions: eux/nous - comme on l'a déjà observé à propos de M. Porete) : ce n'est pas tant que les pratiques religieuses elles-mêmes s'avèrent être des passerelles plutôt que des obstacles, c'est surtout qu'on tend à lire comme des heurts entre communautés ce qui est avant tout la mise en place d'un système de domination adapté aux conditions locales (humaines et écologiques). Par conséquent, ici, les étrangers face auxquels se constituerait une communauté franque ne sont guère qu'un fantasme historiographique - au même titre que celui, signalé antérieurement, qui conduirait à considérer que le discours «patriotique urbain » promu par les autorités urbaines correspondait aux mentalités des populations médiévales.

On voit donc toute la richesse du dossier ici proposé : il permet à la fois de cerner la nature du phénomène communautaire (distingué d'un simple ensemble interpersonnel et polarisé, défini avant tout par ce que les membres ont en commun et secondairement seulement par l'existence d'adversaires communs), de comprendre son articulation avec le système de domination local ou plus large (les logiques communautaires servant à renforcer la domination, au lieu de s'y opposer comme l'ont longtemps affirmé divers historiens ${ }^{21}$ - entre autres en la masquant et/ou en manipulant les démarcations sociales au sein des groupes dominés), enfin de montrer une fois encore combien nous sommes prisonniers non

${ }^{20}$ Reyna Pastor, Del Islam al cristianismo : en las fronteras de dos formaciones económico-sociales, Barcelona, Ed. Península, 1975.

${ }^{21}$ Quelques rappels figurent dans mon article à paraître mentionné supra, n. 10. 
seulement de nos propres représentations sociales, actuelles, mais aussi et surtout de nos croyances à propos de la société médiévale, qui datent, elles, pour l'essentiel du XIX ${ }^{\mathrm{e}}$ siècle. Bref un dossier qui, outre l'intérêt des données factuelles qu'il présente, permet un bon exercice d'hygiène intellectuelle. 